\title{
The Role of Core Strength Training in Sprint Training
}

\section{Zhiquan Tian Feng Dong Jie Yan}

Sports Department, Xi'an University of Technology, Xi'an, Shaanxi, 710048, China

\begin{abstract}
The necessary content of all sports in core strength training, most of its technical movements are through the movement chain constructed by the central muscle group. Strong and powerful central muscle group plays an important role in maintaining the balance of athletes' body posture, motor skills and the stability of special technical movements. The core force is to stabilize the core position between the shoulder and hip joint, control the center of gravity of the body and transfer the strength of the upper and lower limbs.
\end{abstract}

\section{Keywords}

core strength; sprint training; role; strategy

\section{核心力量训练在短跑训练中的作用}

\author{
田质全 董峰 可杰
}

西安理工大学体育部, 中国・陕西 西安 710048

\begin{abstract}
摘 要
核心力量训练时所有运动项目的必要内容, 它的大部分技术动作都要通过中心肌群所构造的运动链。强而有力的中心肌群对 于保持运动员身体姿势的平衡性、运动技能以及专项技术动作的稳定性具有重要作用。核心力量是以稳定人体肩关与髋关节 之间的核心部位为核心，控制身体重心、传递上下肢力量。
\end{abstract}

关键词

核心力量; 短跑训练; 作用; 策略

\section{1 引言}

核心力量的训练对于短跑运动员的专项训练起着至关重 要的作用, 与传统腰腹力量的训练方向不同, 核心力量训练 更加注重深层小肌肉群的训练, 并且训练方式一般为屈伸、 旋转结合起来的复合型、多维度的运动形式, 对全身的运动 都产生着纽带作用。

\section{2 核心力量训练简析}

短跑运动是田径运动中的重要组成部分, 要求运动员用 最短的时间、发挥身体最大的机能跑完固定距离, 在过程中 要将速度提升到极致。短跑运动的主要供能方式为无氧代谢, 对运动员的体能要求较高, 是一种周期性、速度性、力量性 的项目。从一定程度上来讲, 运动员的专项力量素质决定了 跑步质量, 也决定了最终成绩, 它影响着其他素质的构建,

【作者简介】田质全 (1981-), 男, 中国山东菏泽人, 副教授, 硕士研究生学历, 从事体育教育训练学研究。
是短跑运动员必须掌握的训练内容。传统的核心力量素质训 练主要集中在下肢肌群，没有深入研究躯干、髋关节、肩关 节和周边肌群等部位的作用。这种单一化的下肢力量训练无 法为运动员的极限运动快速提供能量, 并维持身体的平衡性。 因此, 认识到核心力量关键训练技术与方法是提高短跑身体 素质的关键手段。

核心力量训练, 简单来说, 就是在训练中通过一定技巧 与身体部位的运用, 以人体的核心区域肌群和神经一肌肉系 统为主, 达到训练身体力量和平衡姿势的训练目的。相较传 统力量训练方式, 核心力量训练更能促进人体核心肌群力量 的提升, 同时还能确保人体在运动中能较好地保持身体的平 衡性、稳定性, 为四肢的发力提供必要支撑, 见表 1 。最初 的核心力量训练只是用在医疗康复领域, 伴随专项运动训练 理论与策略的发展, 越来越多的专业人士将目光投入核心力 量在运动项目训练领域上, 也意识到, 对于任何体育运动来 说，单靠运动员某一个关节或身体的某一部位来完成身体动 
力系统的运行是不现实、不高效的, 这需要发挥运动员身体 各个部位或多个关节共同作用完成的。而运动员短跑质量与 成绩的提升, 同样不能只关注一个方面的训练, 还要将全身 各个关节、各个部位当作一个整体，针对不同的部位加以训 练, 实现整体运动水平与能力的提升。由于短跑是以体能为 主导的速度性项目, 它看重的是运动员的爆发力和身体潜力。 对于短跑运动员来说, 要同时具备力量素质与速度素质, 这 是影响竞技能力的两大要素。由于运动员的先天性因素对速 度素质的影响较大, 对力量素质的影响却较低, 对此, 力量 训练便成为短跑运动员获得优异成绩的必要环节 ${ }^{[1]}$ 。

表 1 测试者核心力量一览表 $(s)(n=5)$

\begin{tabular}{cccc}
\hline & 核心力量缓冲 & 蹬地核心力量 & 离地核心力量值 \\
\hline 王 $* *$ & 0.06 & 0.08 & 0.14 \\
张** & 0.04 & 0.08 & 0.12 \\
贾 ** & 0.06 & 0.06 & 0.12 \\
柴 ** & 0.06 & 0.06 & 0.12 \\
李 ** & 0.04 & 0.06 & 0.1 \\
平均值 & 0.052 & 0.068 & 0.12 \\
标准差 & 0.011 & 0.011 & 0.014 \\
\hline
\end{tabular}

\section{3 核心力量训练在短跑训练中的重要作用}

\section{1 保持身体平衡}

任何运动项目的技术动作都离不开多肌群、多关节、多 部位的协同参与, 一个完整的技术动作需要调动运动员的多 个肌群才能完成。站在动力链的角度而言, 运动员在短跑训 练中, 核心力量训练主要能发挥传导发力、稳定脊柱与骨盆、 维持身体姿势的平衡性、提高肌能的稳定能力等作用。此外, 核心量训练是人体进行全身发力的起始点, 它能维持上下肢 的协调性, 同时还能促进能量的传递。短跑的核心技术动作 是奔跑过程中两大腿的快速剪绞, 影响着短跑的速度, 而人 体核心区, 如髋关节的稳定性与灵活性则能有效减少两大腿 的剪绞与制动时间。简单来说, 当增加了髋关节的关节活动 幅度, 那么就等同于增加了大腿的摆动幅度, 意味着速度的 增加。伴随盆骨周围肌群力量的增强, 在跑步过程中也能显 著提高其稳定性, 快速回复身体重心, 减少重心的变化幅度, 让运动员保持线性运动状态, 对于提高运动员的极限运动速
度是极其有利的 ${ }^{[2]}$ 。

\section{2 预防运动损伤}

导致短跑运动员发生损伤的因素多种多样, 主要包括准 备工作不充分、长期性运动疲劳、技术动作不㜀熟、运动后 不注意放松、肌体恢复能力差、极限供能不足、躯干支柱力 量薄弱等。短跑运动的力量往往需要经过核心区传递到四肢, 如果核心力量运用不足, 特别是当髋关节的不稳定的时候, 人体内的能量会在髋关节快速流失, 身体便会出现代偿动作。 久而久之, 短跑运动员发生伤病的次数便会增多, 这便是很 多运动员形成旧伤而难以养好的主要原因。对于高水平运动 员来说, 他们的大肌肉块具有较强的力量, 而小肌肉的力量 相对薄弱。小肌肉的稳定性又关系着运动员肢体的稳定性, 如果没有科学锻炼小肌肉的力量, 可能会出现急性损伤现象。 如果发力不正确, 还会直接降低跑步质量。核心力量训练要 以整体训练为主, 应当具备完整的训练计划, 切实保障运动 员的关节功能, 优化身体平衡度、灵活度, 改善身体形态, 让关节运动更加灵活、高效。在日常训练中, 要侧重于强化 核心区、躯干的力量训练，只有在保证身体的稳定性之后， 才能发挥技术动作的重要作用, 降低身体耗能, 减轻腰部与 膝关节的压力, 避免发生运动损伤 ${ }^{[3]}$ 。

\section{3 作用短跑过程}

核心力量的训练除了具有上述两个作用之外, 对于短跑 过程中也具有较大的作用。一是加速起跑, 传递力量。当短 跑运动员熟练掌握相关训练技巧并能有效运用在比赛过程中, 那么便可以促进核心区的力量更好地传递到四肢，充分调动 身体的各个部位、各个关节, 组建一条完整的运动链, 在起 跑冲刺中发挥踝、膝、髋、腰等部位的力量作用, 从而缩短 起跑时间, 达到加速起跑的目的。短跑的起跑动作是两首快 速推离地面, 并使用两臂屈肘有力地作前后摆动, 两只腿同 时用力蹬起跑器, 前腿以膝关节为中心摆动。起跑时, 要特 别注意对中心前移、平衡控制等技术的运用, 这些都要依靠 四肢的协调作用, 核心力量的训练则可以发挥承上启下的作 用, 为四肢传输更强的力量。

第一，起跑时人体重心前移，核心力量能在维持姿势平 衡的前提下作出冲刺状态，促进以髋为轴的高速提脚、摆动 动作, 这是提高短跑成绩的根本途径。二是提速途中跑, 完 善技术。针对肌肉群的训练, 能让短跑加速跑更加省力, 达 到提速途中跑的目标。 
第二，短跑技术中的 “蹬摆结合” 提升了大腿以髋关节 为轴的摆动效率, 也更加重视对核心力量的应用。以核心区 耻骨为中心点形成中心线、髋关节为轴的横轴面形成圆周, 在短跑训练中保持身体左右的移动平稳性, 便于大腿前后摆 动形成弧形落地, 膝关节也控制在圆周运动范围内, 通过这 一方式完善力量的速度。在途中跑中要注重身体姿势的完整 性, 特别是核心区域的髋关节控制能力、腿肌的运用都要达 到最完善的分配与互补; 运动员要放松上半身, 以髋带膝、 以膝带动脚，这是短跑过程中提速的最理想方式。

第三, 帮助后程冲刺, 稳定核心。对于短跑运动来说, 最为关键的便是后程冲刺技术的运用, 它与途中跑相似, 只 是在后程跑中, 由于经过加速跑的极限耗能过程, 运动员的 身体会出现疲劳感, 而为了保持速度, 还需要加强后蹬和双 臂摆动, 在距离终点线的最后一步时, 要快速前倾上身或肩膀, 用胸部、肩膀等部位触碰终点线并不断减缓速度。在整个过 程中, 对人体能量的需求都是极大的。因此, 增强短时间能 量的供应能力成为主要问题, 而磷肌酸或乳酸可以在短时间 内提供能量, 是短跑运动员不可缺少的系统。它们就如 ATP 一样被存储在肌肉当中, 对力量训练产生着重要作用。

为了保持工作肌肉群的稳定性, 单单进行跑步训练是完 全不够的, 对肌肉力量进行训练, 能让肌肉始终保持强壮, 并且髋关节肌肉群能有效控制四肢的摆动作用, 快速增加速 度, 加强四肢协调与力量的传递作用。力量训练还可以防止 身体损伤, 让核心关节保持稳定状态。核心力量训练可以为 后程冲击提供充足的能量, 增强运动效率 ${ }^{[4]}$ 。

\section{4 核心力量训练在短跑训练中的应用策略}

\section{1 训练原则}

在短跑运动的核心力量训练过程中, 要遵循一定的原则, 并且还要结合运动员自身情况、实际水平等, 循序渐进地设 置训练内容。首先, 在基础训练阶段, 应当注重自重训练法, 即感知受脊柱的力量和骨盆的稳定性。同时可以应用平衡球、 瑜伽球等物体增强身体的平衡性, 让运动员掌握力量传递的 有效方法。其次, 在技能提升阶段, 要进行核心区的力量训练, 即腰胯摆动力, 同时训练大腿肌肉和韧带的伸展性、弹性, 让各关节保持灵活性。最后, 在专项训练保持阶段, 要重视 核心速度力量以及爆发力的训练，增加胯关节的摆动速度以
及摆动幅度，结合运动特性开展针对性训练活动。

\section{2 训练方法}

在稳定条件下, 可以一边维持原有姿势, 一边进行可控 运动。该训练方法是针对短跑运动员的核心力量初期训练阶 段, 此阶段的训练强度属于中小型, 关键在于刺激核心肌群 表层肌肉的本体感觉, 增强人体重要肌肉的力量以及耐力, 下面将提出三种合适的训练方法。

其一, 对角俯卧支撑。首先要从标准俯卧撑的起始姿势 开始, 抬起左腿同时向前伸出右臂, 左腿与右臂都要保持平 直状态。头部抬起, 将身体舒展到最大, 要坚持尽可能长的 时间，然后再换对侧手臂与腿，不断重复练习。

其二, 站姿绳索抗旋转。此方法从站姿开始, 双脚距离 比肩宽，抬头挺胸面向前方，伸出双手抓住绳索，然后将绳 索向左边拉动, 整个过程中都不能移动躯干, 重点感知核心 肌群的发力。

其三，仰卧提腿。运动员仰卧，将手放在臀部下方， 头微微上台, 脚踝伸直, 脚跟立地 $15 \mathrm{~cm}$, 膝盖不能弯曲, 缓慢提高双腿至 $45^{\circ}$, 保持 $3 \mathrm{~s}$ 后放下，重复做 10 次，每次 5 组 ${ }^{[5]}$ 。

在非稳定条件下，前提是要控制好身体重心，以便为躯 体的运动训练创造支撑点, 可以提高核心区、肩关节的力量, 让跟股东的肌肉群控制躯干。练习时可使用气垫、平衡盘、 悬吊等。单腿撑地收腹。仰卧, 将腰放置在瑞士球上, 双手 虚扶头两侧, 左腿撑地, 右腿放在左膝盖上。用腹直肌发力 进行缓缓收缩, 下落时可适当伸展, 整个过程都要保持稳定性。 重复 20 次换右腿撑地循环练习。

\section{参考文献}

[1] 唐斯英. 浅谈短跑运动员核心力量的训练方法 [J]. 青少年体 育, 2017(02):52-52+102.

[2] 王高阳. 短跑运动员的核心力量训练研究 [J]. 体育世界 (学术 版),2016(12):92-93.

[3] 赵俊华, 周玉斌, 张成. 对短跑运动员进行身体核心区力量训练的 实验研究 [J]. 北京体育大学学报 ,2015(06):165-166.

[4] 陈振伦. 短跑运动员基础力量的核心力量训练实验研究 [J]. 哈尔 滨体育学院学报,2014(05):48-49.

[5] 戴绍斌. 核心力量训练在田径项目中的运用研究 [J]. 三峡大学体 育学院 ,2017(24):23-24. 